So the muscles of the abdominal wall vary and so the operation of lumbar colotomy as performed in $\mathrm{my}$ presence by a professor was one of the most gruesome sights I ever saw in my life. He divided a lumbar artery with its accompanying nerve-he carefully identified them but could not tie the artery, for he lost it in the wealth of muscular tissue which he unravelled, and after her death his patient was found to have her peritoneal cavity full of blood. I do not recollect ever having a fatal colotomyI am certain no case of mine ever died from hæmorrhage, for I disregard all anatomical curiosity during the operation. I put the patient on her right side with her knees bent up to steady her and I place a pillow under her right loin so as to put on the stretch that which is the guide to the whole thing - a strip of tendon which marks the union of the fascias encasing the external oblique muscle, the internal oblique, the transversalis on the one side and the latissimus dorsi (or its tendon) and the quadratus lumborum on the other. The real difficulty is that the relations of the edges of these muscles do not follow the directions of the text-books as of course they ought to do. Let the operator disregard the text-books and bear in mind that after he has divided the skin to a necessary extent over the tight band and absolutely in its direction he has only to open the glistening fascia in the same position and carefally look at the direction of the muscular fibres with which he meets. He must in his proceedings inwards bear only one route in mind and that is as nearly as possible to the umbilicus. Only one direction further is necessary so far as muscular fibres are concerned, that the surgeon must put his knife down and use only his blunt forefingers to separate the fibres. At first this would be in a direction parallel to the axis of the patient's trank and afterwards at right angles to that axis-he need not trouble to identify even the muscles. When he has got through the two layers he will come on a layer of fascia of the greatest importance, concerning which he ought to be "well up"-the transversalis fascia. This he must slightly notch and after getting his forefingers through it he must search backward towards the body of the vertebra till he finds the base of the kidney. Having found this and remembering two arid facts of anatomy-that the descending colon is generally immediately in front of the kidney if placed on its usual side, in front of his fingers, is attached to the moveable peritoneum and separated from his finger by some loose fat, he will take two or three pairs of catch forceps and pull layer after layer of fat boldly and freely up into the wound, leaving the forceps on each layer as he lands it outside, and proceed in this way till he sees the unmistakeable gut. If be keeps the points of his forceps well downwards and pointing well forwards towards the umbilicus he cannot miss the gut. When the gat is missed it is always by dissecting-room people who go over it instead of at it. Having found the gut, haul it freely and fearlessly up out of the wound, cut it nearly through and stitch with an all-round suture into the lower angle of the wound, carefully replacing all fringes of fat as you proceed, or removing them if much injured by the forceps, but remove them with your fingers and not with cutting instruments. You then close the rest of the wound, the upper part. The wound need never be more than three inches long and generally need not be so much, but it may look like five inches deep. A wound so made heals immediately, and as there is nothing but a temporary separation of muscular fibres they fall back at once into position and at once resume their function. The whole gut has been pulled through the crosswise-made opening in the undamaged muscle, so that the almost constant tension involved in all active positions of the body secures the closure of the aperture in the gut. In a badly done colotomy only a bit of the circumference of the gut is pulled up to or out of the muscular aperture, the bulk of it being as before left inside and the gut is emptied through the relief passage only by regargitation from below and generally when the whole of it is distended by hardened fæces. This does not give full or indeed much relief, the retained fæces having from time to time to be dug out. After colotomy is done as I recommend it should be done all the motion comes through the wound at once and after some practice there comes marvellous control over the passage.

If anyone compares the proceedings described above with those of Amussat as described by himself and as copied or altered in text-book after text-book after him, it will be seen that there is very little in common between the two. The operation as I give it I got from the lips of Alexander
McKenzie Kldwards, lecturer on surgery in the Extra-Mural School of Edinburgh, to whom I was apprentice, pupil, and assistant for about six years. I have no recollection of hearing from him that he got it from anybody else and I certainly have neither read nor heard of it elsewhere. He was a surgical genius of the highest order, as many can testify who are still alive who knew him, so that I have no doubt it was a conception of his own, and if any name is to be attached to it his name alone ought to be.

When the time came in the "seventies" that I ventured to operate upon and to remove kidneys I adopted the same incision save in such cases as those where I followed another rule as simple and imperative. Attack your tumour on its most prominent aspect and certainly avoid all dissectingroom rules about finding "oblique lines" or other untrust-. worthy directions.

Birmingham.

\section{A NOTE ON THE ETIOLOGY OF BERI-BERI.}

BY WALTER K. HUNTER, M.D., B.Sc. GLASG., ASSISTANT PHYSTCIAN TO THE ROYAL INFIRMARY AND TO THE ROYAI HOSPITAL FOR SICK CHILDREN, GLASGOW.

IN THE LANCET of July 31st, 1897, I reported the results. of a bacteriological examination of the blood in two cases of beri-beri. Since then I have had the opportunity of carrying. out in two other cases a similar investigation, and this I now put on record in further support of the opinion already expressed-namely, that the white staphylococcus of Pekelharing and Winkler is in trath the specific micro-organism of beri-beri. The two patients, both seamen from the s.s. City. of London, were admitted into Dr. Middleton's wards of the Glasgow Royal Infirmary on Oct. 29th, 1897. Neither of them spoke English with any degree of fluency and so the account of their illnesses is necessarily very brief.

CASE 1. -The patient was a native of Bombay and as faras could be made out his illness began fourteen days previously to admission with swelling and pain in the feet and legs. There was considerable diffculty in walking and the patient was sick and very drowsy. On admission to the wards the cedema of the feet and legs was well marked. The sensation to touch and pain seemed to be blunted over. a considerable extent of the body, but examination on this point was not very satisfactory. The superficial reflexes were quite active. The left knee reflex was absent and the right was diminished. The patient walked fairly well except. when he was told to go along a straight line and then he had a tendency to stagger. He could not stand with the feet close together and the eyes shut. No reaction of degeneration was found in any of the muscles. The right ventricle of the heart seemed to be somewhat dilated, but no. murmur was to be heard over any part of the cardiac area. There was evidence of some fluid effusion into both pleura cavities. The urine was quite normal. Fxamination of the blood gave the following results : hæmoglobin, 70 per cent. red corpuscles, 71.5 per cent.; and white corpuscles, 1 in 450 . The patient remained in hospital for five weeks and during that time the only change noted was a return to activity of both knee reflexes. At no time was albumin to be found in the urine.

CASE 2.-The patient was a native of Zanzibar and the history of his illness was very similar to that in Case 1. He had swelling and pain in the feet and legs, but there was no sickness or difficulty in walking. $\mathrm{He}$ could stand with the eyes shut and the feet close together without any difficulty. The superficial reflexes were present, but both knee reflexes were abolished. The sensation to pain seemed to be practically absent all over the body and the sensation to touch was much impaired. There was no reaction of degeneration in any of the muscles. The lungs were quite healthy, but there was a suspicion of some mitral incompetence. The urine was perfectly normal. Examination of the blood gave the following results: hæmoglobin, 90 per cent. ; red corpuscles, 85 per cent.; no increase of white corpuscles. The patient was dismissed from the hospital after a month's residence in much the same condition as on admission, the knee reflexes being still absent and sensation in no way improved.

In both these cases specimens of freshly. drawn blood were examined at varions times and on each occasion microorganisms were to be seen in rapid motion in the spaces 
Detween the groups of corpuscles. Culture media were inoculated with the blood as follows: on Oct. 31st 4 agar-agar and 1 bouillon tubes from each case; on Nov, 10 th 3 agar-agar and 3 bouillon tubes from each case; and on Nov. 23rd 4 bouillon tubes from each case. Of the .30 tubes thus inoculated growth was obtained in only 4, 3 of these having been inoculated from Case 1 and the fourth from Case 2. Of these 4 growths, 3 of them (2 from Case 1 and 1 from Case 2) were pure, and they presented all the characters of the staphylococcus beri-beri as already deseribed in my former paper. They liquefied gelatin in three weeks. The fourth growth (from Case 1) was impure, consisting chiefly of streptococci, but from it no staphylococcus could be isolated.

The pathogenic properties of these staphylococcus growths were investigated by injecting the organisms grown in bouillon broth into rabbits.

Rabbit 1 was injected with staphylococcus grown from Case 1. In this rabbit 8 injections (each injection consisting of $10 \mathrm{c.mm}{ }^{1}$ of a forty-eight hours' bouillon growth), extending over 18 days-i.e., an injection every second day-were given before signs of paralysis showed themselves. The post-mortem examination of the rabbit was quite negative as regards naked-eye appearances, there being no signs whatever of peritonitis and no abscesses in the liver. The nerves, however, showed unmistakeable evidence of parenchymatous degeneration. The experiment, then, serves to establish the xelationship of this staphylococcus with the staphylococcus beri-beri of Pekelharing and Winkler and at the same time it demonstrates that it is not staphylococcus pyogenes albusmot at least as one usually meets with that micro-organism.

The next two rabbits were injected with growths of the staphylococcus which had been subjected for twenty-four hours to a temperature of $41^{\circ} \mathrm{C}$.

Rabbit 2 (injected with growth from Case 1) and Rabbit 3 (injected with growth from Case 2) each received twelve injections which extended over four weeks, each injection containing 10 c.mm, of a bouillon broth culture. That the virulence of these growths had been lessened by the tempera*ure they had been submitted to seems to be undoubted, for at the end of the experiment the animals looked as healthy as at the commencement. After an interval of ten days the same rabbits were injected with non-attenuated growthsi.e., with the same growths as those which killed Rabbit 1. Thach rabbit received nine injections, each of $12 \mathrm{c.mm}$., extending over twenty-one days. At the end of this time the rabbits still showed no signs of paralysis. They were then killed, but the post-mortem appearances in both were again negative. The white staphylococcus was cultivated from the heart and spleen in both. Careful microscopic examination of the nerves showed undoubtedly that fibres here and there were undergoing parenchymatous degeneration, but the degeneration was not nearly so marked as in Rabbit 1. Thus it is seen that in rabbits of the same size the two previously injected with an attenuated micro-organism seemed Hess easily affected by the unattenuated organism than those which were not so injected. I scarcely wish to suggest that an immunity bad been produced, for a conclusion "sannot be drawn from solitary observations. But the experiments at least make one think of the possibility of producing an immunity or of lessening the susceptibility to the poison of beri-beri.

As regards the source of infection of the two patients I was supplied through the kindness of Dr. George Marshall with samples of their food. These samples consisted of rice, isplit peas, and two kinds of dried fish. Several cultures were taken from each, with the result that from the rice there was readily cultivated a white staphylococcus with : similar morphological characters to the staphylococcus beriberi which was grown from the blood of the two patients. 'This staphylococcus which was grown from rice liquefied igelatin in 19 days. Its pathogenic properties were tested by injecting a fourth rabbit.

Rabbit 4 was injected with bouillon culture which was grown from rice; 12 injections, each consisting of $10 \mathrm{c} . \mathrm{mm}$. of a forty-eight hours' bouillon growth were given, extending over four weeks. At the end of that time no signs of paralysis had presented themselves, so the animal was killed. "The result of the post-mortem examination as regards nakedeye appearances was quite negative. The nerves, bowever,

1 An error occurs throughout my former paper, where it is stated that cubic centimetres (c.c.) of the bouillon broth were injected into the vabbits. It was, of course, cubic millimetres (c.mm.) which were injected. on microscopic examination showed unmistakeable signs in many places of a parenchymatous degeneration. Cultures of the white staphylococcus were readily grown from the blood of this rabbit.

I conclude, then, from the above that this white staphylococcus which was grown from rice is the same microorganism as that which was found in the blood of the two patients and therefore $I$ consider rice taken as food may be a source of infection. Possibly, however, it may be that rice only makes a suitable medium for the growth of the staphylococcus and that the entrance of the infection is entirely through the air passages. If one may draw any conclusion from comparing the experiment of Rabbit 1 with that of Rabbit 4 it would be that the staphylococcus grown on rice is less virulent than when grown from the human blood. This, however, is not warranted, for the data are too uncertain. The white staphylococcus grown from rice was not found in any of the growths from the other specimens of food.

I again have to express my great indebtedness to Dr. G. S. Middleton, who so kindly placed at my disposal these two cases of beri-beri. I have also to thank Dr. Lindsay Steven and Dr. J. Barlow for the use of their laboratories and apparatus.

Glasgow.

\section{A CASE OF FRACTURED SPINE WITH SOME UNUSUAL COMPLICATIONS ; RECOVERY.}

BY A. W. LYONS, M.B. LOND., M.R.C.S. ENG., HONORARY MEDICAL OFFICER TO THE TEAMES DITTON COTTAGE HOSPITAL.

ON Dec. 24th, 1897, a carter, aged twenty-eight years, was leading his horse, which was attached to an empty brickcart, under a railway-bridge when the horse took fright at a passing train, bolted, and knocked him down, causing the cart-wheel to pass over his back. I found the patient lying by the side of the road, whither he had been carried. $\mathrm{He}$ complained of great pain in his back, and he was bathed in a profuse perspiration. His pulse was feeble; 65 per minute. He was conveyed to the Thames Ditton Cottage Hospital on an ambulance. On admission no bruises or other external marks of injury were discovered, but there was a puffy swelling over the situation of the ninth and tenth dorsal vertebræ. On palpating this the finger appeared to sink into the swelling; but it was difficult to make out the vertebral spines though the one next above the seat of injury appeared to be a little prominent. No crepitus was made ont, neither was there any distinct dislocation of the spine. A carefal search was made for a fracture of the ribs but none was discovered. At this time there was no marked paralysis, though the movements of both legs were feeble, especially of the right. The patellar reflexes were both absent. The patient was laid flat on his back and was kept in position by means of a folded towel placed across his chest and secured at the ends by a sandbag on either side. The urine was drawn off and was found to be normal. By the evening of the same day the right leg was paralysed, the patient being quite unable to draw it up in bed though he could still move the foot a little. The movements of the left leg were impaired considerably more than they had been in the morning. The patellar reflexes were both absent. He had been coughing a little and bringing up some blood-stained mucus. On auscultation a few râles were heard over the right lung but none over the left lang. Resonance was equal on both sides. The temperature of the patient on admission was $98.2 \mathrm{~F}$. In the evening of the same day it was $99^{\circ}$. On Dec. 25th the temperature was $99 \cdot 4^{\circ}$. The patient had passed a fairly good night, but the cough had been a little troublesome. Bloodstained mucus was still being brought up. The cough caused him acute pain over the seat of injury to his back. The movements of the right side of the chest were much diminished and there was dulness over the whole of that side. The apex beat was not displaced. Vocal fremitus was absent and no tubular breathing could be heard, but the breath sounds were very faint and vocal resonance was much diminished. The patient complained of shooting pains in the right leg. The paralysis remained stationary and there was still retention of urine. There had been no action of 